\title{
Optimal placement of distributed generation in distribution networks
}

\author{
Satish Kansal $^{1 *}$, B.B.R. Sai ${ }^{2}$, Barjeev Tyagi ${ }^{3}$, Vishal Kumar ${ }^{4}$ \\ Department of Electrical Engineering, Indian Institute of Technology, Roorkee, INDIA \\ *Corresponding Author: e-mail:kansal.bhsb@gmail.com, Tel+91-1332-284331, Fax+91-1332-273560
}

\begin{abstract}
This paper proposes the application of Particle Swarm Optimization (PSO) technique to find the optimal size and optimum location for the placement of DG in the radial distribution networks for active power compensation by reduction in real power losses and enhancement in voltage profile. In the first segment, the optimal size of DG is calculated at each bus using the exact loss formula and in the second segment the optimal location of DG is found by using the loss sensitivity factor. The analytical expression is based on exact loss formula. The optimal size of DG is calculated at each bus using the exact loss formula and the optimal location of DG is found by using the loss sensitivity factor. The proposed technique is tested on standard 33-bus test system and the obtained results are compared with the exhaustive load flows.
\end{abstract}

Keywords: distributed generation, Particle Swarm Optimization (PSO), optimal size, optimal location, power loss.

\section{Introduction}

The objective of power system operation is to meet the demand at all the locations within power network as economically and reliably as possible. The traditional electric power generation systems utilize the conventional energy resources, such as fossil fuels, hydro, nuclear etc. for electricity generation. The operation of such traditional generation systems is based on centralized control utility generators, delivering power through an extensive transmission and distribution system, to meet the given demands of widely dispersed users. Nowadays, the justification for the large central-station plants is weakening due to depleting conventional resources, increased transmission and distribution costs, deregulation trends, heightened environmental concerns, and technological advancements. Distributed Generations (DGs), a term commonly used for small-scale generations, offer solution to many of these new challenges. CIGRE define DG as the generation, which has the characteristics (CIGRE, 1999): it is not centrally planned; it is not centrally dispatched at present; it is usually connected to the distribution networks; it is smaller then 50100MW. Other organization like, Electric Power Research Institute define distributed generation as generation from few kilowatts up to 50MW. Ackermann et al. have given the most recent definition of DG as: "DG is an electric power generation source connected directly to the distribution network or on the customer side of the meter." Using DG can enhance the performance of a power system in many aspects. Employing DG in a distribution network has several advantages as (Khoa et al, 2006), reduction in line losses, emission pollutants, overall costs due to improved efficiency \& peak saving. Improvement of voltage profile, power quality, system reliability and security and the disadvantages are (Illerhsus et al, 2000), reverse power flow, injected harmonics, Increased fault currents depending on the location of DG units. DG also has several benefits like energy costs through combined heat and power generation, avoiding electricity transmission costs and less exposure to price volatility (Ghosh et al, 2010).

\section{Location and Sizing issues}

Fig. 1 shows a 3D plot of typical power loss versus size of DG at each bus in a standard 69-bus distribution test system. From the figure, it is obvious that for a particular bus, as the size of DG is increased, the losses are reduced to a minimum value and increased beyond a size of DG (i.e. the optimal DG size) at that location. If the size of DG is further increased, the losses starts to increase and it is likely that it may overshoot the losses of the base case. Also notice that location of DG plays an important role in minimizing the losses. The important conclusion that can be drawn from Fig. 1 is that, given the characteristics of the distribution 
system, it is not advisable to construct sufficiently high DG in the network. The size at most should be such that it is consumable within the distribution substation boundary. Any attempt to install high capacity DG with the purpose of exporting power beyond the substation (reverse flow of power though distribution substation), will lead to very high losses (Lakshami et al, 2008). So, the size of distribution system in term of load (MW) will play important role is selecting the size of DG. The reason for higher losses and high capacity of DG can be explained by the fact that the distribution system was initially designed such that power flows from the sending end (source substation) to the load and conductor sizes are gradually decreased from the substation to consumer point. Thus without reinforcement of the system, the use of high capacity DG will lead to excessive power flow through smallsized conductors and hence results in higher losses.

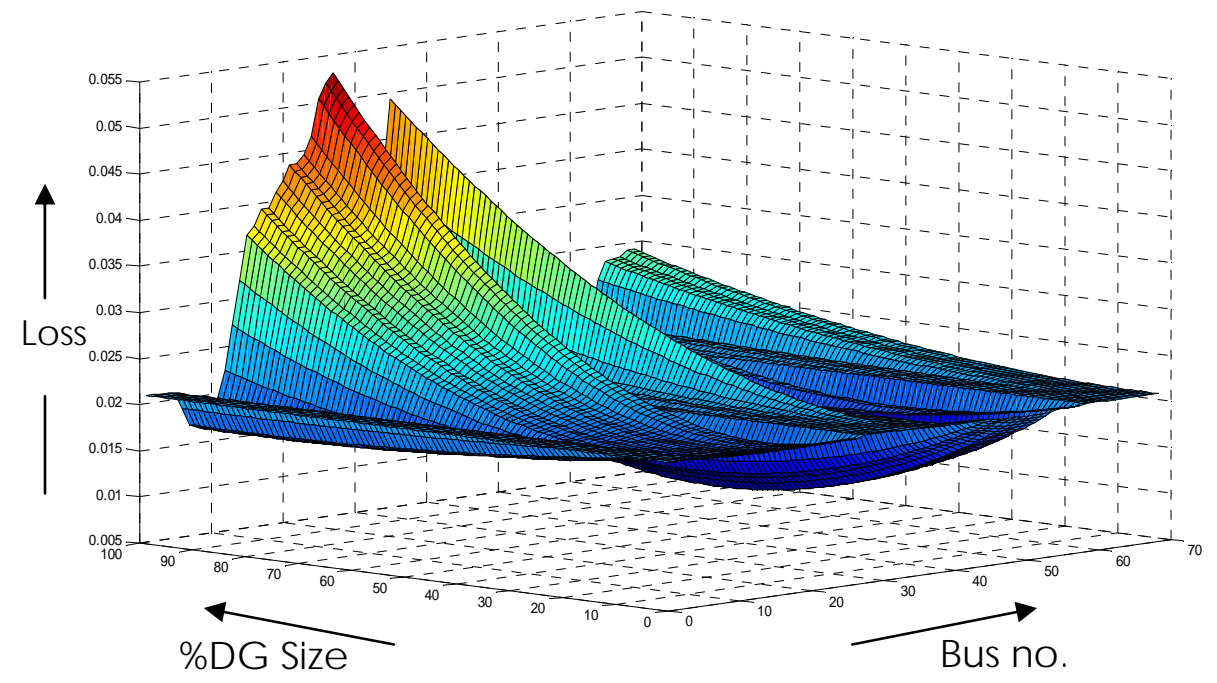

Figure 1. Effect of size and location of DG on system loss.

\subsection{Loss sensitivity factor:}

The loss sensitivity factor is used for the placement of DG is explained as, the real power loss in the system is given by (1).This formula is popularly referred as "Exact Loss" formula (Elgerd, 1971; Kazemi et al, 2009).

$P_{L}=\sum_{i=1}^{N} \sum_{j=1}^{N}\left[\alpha_{i j}\left(P_{i} P_{j}+Q_{i} Q_{j}\right)+\beta_{i j}\left(Q_{i} P_{j}+P_{i} Q_{j}\right)\right]$

Where,

$$
\begin{aligned}
& \alpha_{i j}=\frac{r_{i j}}{v_{i} v_{j}} \operatorname{Cos}\left(\delta_{i}-\delta_{j}\right) \\
& \beta_{i j}=\frac{r_{i j}}{v_{i} v_{j}} \operatorname{Sin}\left(\delta_{i}-\delta_{j}\right)
\end{aligned}
$$

and

$z_{i j}=r_{i j}+j x_{i j}$ are the $\mathrm{ij}^{\text {th }}$ element of [Zbus] matrix

$$
\mathrm{P}_{\mathrm{i}}=\mathrm{P}_{\mathrm{Gi}}-\mathrm{P}_{\mathrm{Di}} \quad \text { and } \quad \mathrm{Q}_{\mathrm{i}}=\mathrm{Q}_{\mathrm{Gi}}-\mathrm{Q}_{\mathrm{Di}}
$$

$\mathrm{P}_{\mathrm{Gi}} \& \mathrm{Q}_{\mathrm{Gi}}$ are power injection of generators to the bus.

$P_{D i} \& Q_{D i}$ are the loads. 
$P_{i} \& Q_{i}$ are active and reactive power of the buses.

The sensitivity factor of real power loss with respect to real power injection from the DG is given by

$\alpha_{i}=\frac{\partial P_{L}}{\partial P_{i}}=2 \alpha_{i i} P_{i}+2 \sum_{\substack{j=1 \\ j \neq i}}^{N}\left(\alpha_{i j} P_{j}-\beta_{i j} Q_{j}\right)$

Sensitivity factor are evaluated at each bus by using the values obtained from the base case load flow. The bus having lowest loss sensitivity factor will be best location for the placement of DG (Acharya et al, 2006). Conventional load flow studies like Gaussseidal, Newton raphson and fast decoupled load flow methods are not suitable for distribution load flows because of high R/X ratio. A load flow method for distribution systems i.e backward sweep and forward sweep method for load flow that offers better solution was proposed (Haque 1996).

\subsection{Optimal Sizing of DG:}

The total power loss against injected power is a parabolic function and at minimum losses, the rate of change of losses with respect to injected power becomes zero [9].

$\frac{\partial P_{L}}{\partial P_{i}}=2 \alpha_{i i} P_{i}+2 \sum_{\substack{j=1 \\ j \neq i}}^{N}\left(\alpha_{i j} P_{j}-\beta_{i j} Q_{j}\right)=0$

It follows that

$P_{i}=\frac{1}{\alpha_{i i}}\left[\sum_{\substack{j=1 \\ j \neq i}}^{N}\left(\alpha_{i j} P_{j}-\beta_{i j} Q_{j}\right)\right]$

Where $\mathrm{P}_{\mathrm{i}}$ is the real power injection at node $\mathrm{i}$, which is the difference between real power generation and the real power demand at that node:

$P_{i}=\left(P_{D C i}-P_{D i}\right)$

Where $\mathrm{P}_{\mathrm{DGi}}$ is the real power injection from DG placed at node $\mathrm{i}$, and $\mathrm{P}_{\mathrm{Di}}$ is the load demand at node $\mathrm{i}$. By combining the above we get.

$P_{D G i}=P_{D i}-\frac{1}{\alpha_{i i}}\left[\sum_{\substack{j=1 \\ j \neq i}}^{N}\left(\alpha_{i j} P_{j}-\beta_{i j} Q_{j}\right)\right]$

The equation (5) gives the optimum size of DG for each bus $i$, for the loss to be minimum. Any size of DG other than $P_{D G i}$ placed at bus $i$, will lead to higher loss. 


\subsection{Optimal Location of DG:}

The optimal location can be find for the placement of optimal sizes of DG as shown in fig.(2) as obtained from eq. (5) which will give the lowest possible total loss due to placement of DG at the respective bus is as shown in fig. (3). The bus having least power loss will be optimal location for the placement of DG (Acharya et al, 2006).

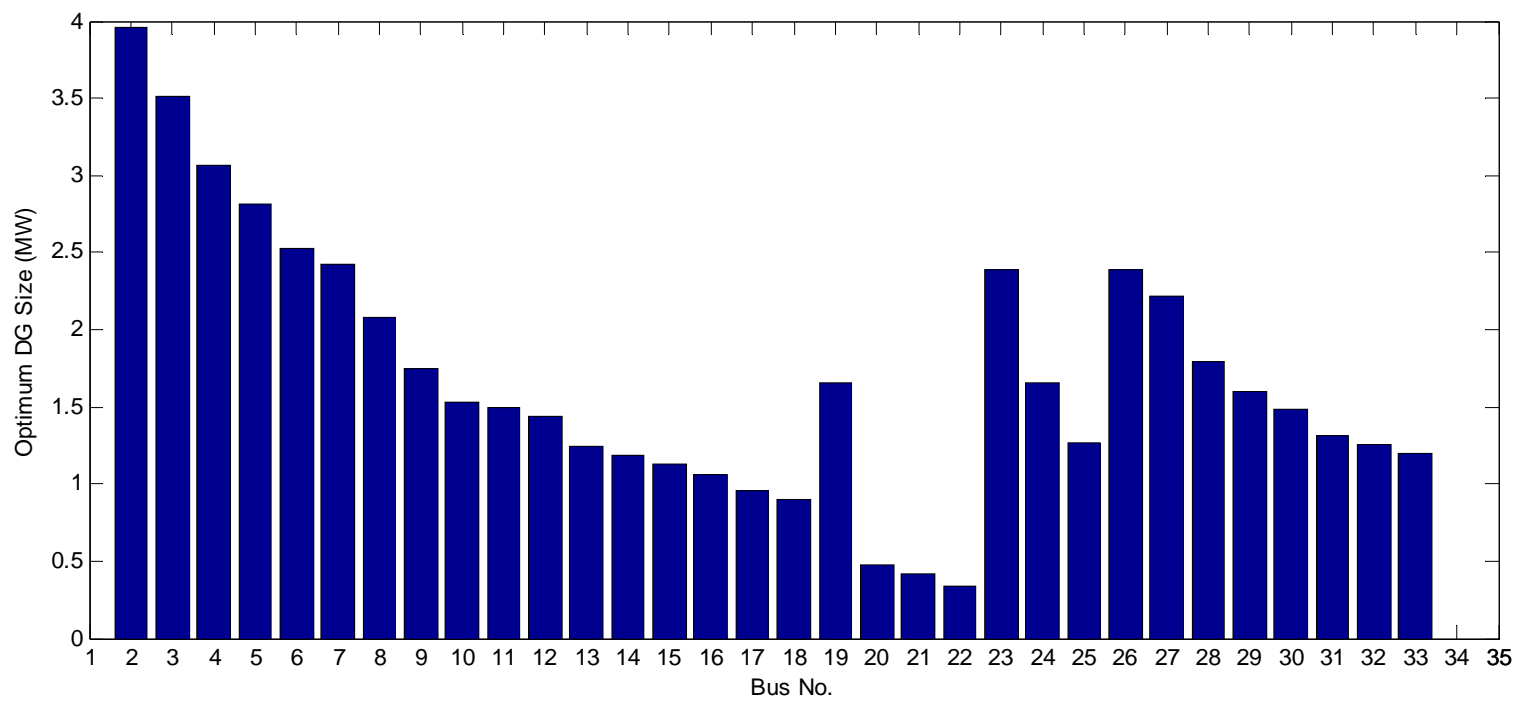

Figure 2. Optimum size of DG at various locations for 33 bus distribution system

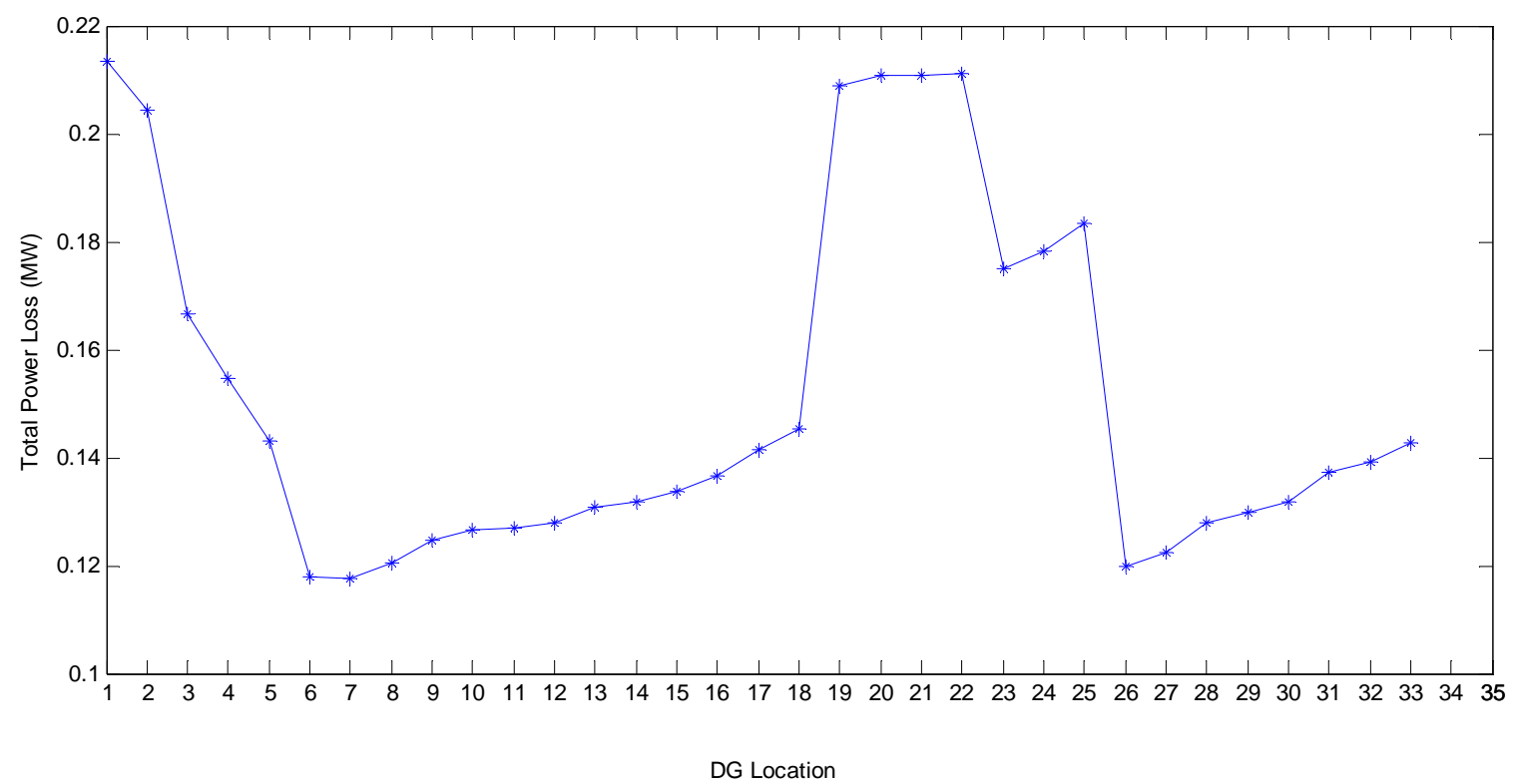

Figure 3. Accurate Total Power Loss of 33 bus distribution system. 


\section{Particle Swarm Optimization}

\subsection{Introduction}

Particle swarm optimization (PSO) is a population-based optimization method first proposed by Kennedy and Eberhart in 1995, inspired by social behavior of bird flocking or fish schooling (Kennedy et al, 1995). The PSO as an optimization tool provides a population-based search procedure in which individuals called particles change their position (state) with time. In a PSO system, particles fly around in a multidimensional search space. During flight, each particle adjusts its position according to its own experience (This value is called Pbest), and according to the experience of a neighboring particle (This value is called Gbest), made use of the best position encountered by itself and its neighbor (Fig 4).

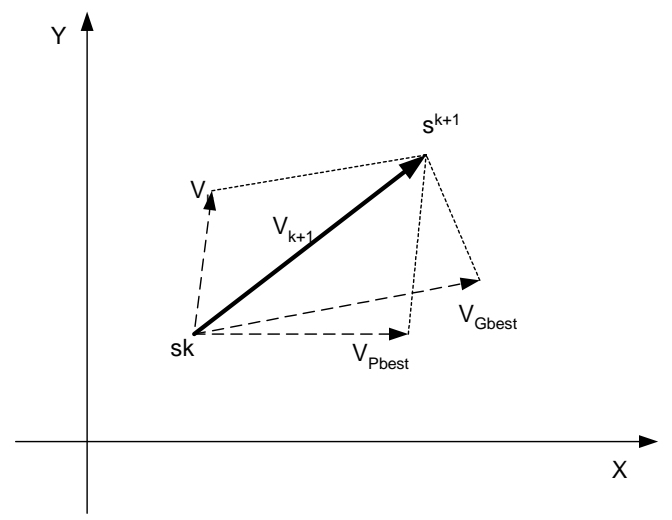

Figure 4. Concept of a searching point by PSO

This modification can be represented by the concept of velocity. Velocity of each agent can be modified by the following equation:

$$
v_{i d}^{k+1}=\omega v_{i d}^{k}+c_{1} \text { rand } \times\left(\text { pbest }_{i d}-s_{i d}^{k}\right)+c_{2} \text { rand } \times\left(\text { gbest }_{i d}-s_{i d}^{k}\right)
$$

Using the above equation, a certain velocity, which gradually gets close to pbest and gbest can be calculated. The current position (searching point in the solution space) can be modified by the following equation:

$$
\begin{gathered}
s_{i d}^{k+1}=s_{i d}^{k}+v_{i d}^{k+1}, i=1,2, \ldots, n . \\
\quad d=1,2, \ldots, m
\end{gathered}
$$

Where,

$s^{k}$ is current searching point,

$s^{k+1}$ is modified searching point,

$\mathrm{v}^{\mathrm{k}}$ is current velocity,

$\mathrm{v}^{\mathrm{k}+1}$ is modified velocity of agent $i$,

$v_{p b e s t}$ is velocity based on pbest, ,

$v_{\text {gbest }}$ is velocity based on gbest,

$n$ is number of particles in a group,

$m$ is number of members in a particle,

pbest $_{\mathrm{i}}$ is pbest of agent $i$,

gbest $_{\mathrm{i}}$ is gbest of the group,

$\omega_{i}$ is weight function for velocity of agent $i$,

$c_{i}$ is weight coefficients for each term.

The following weight function is used:

$$
\omega_{i}=\omega_{\max }-\frac{\omega_{\max }-\omega_{\min }}{k_{\max }} . k
$$


Where,

$\omega_{\min }$ and $\omega_{\max }$ are the minimum and maximum weights respectively. $k$ and $k_{\max }$ are the current and maximum iteration. Appropriate value ranges for $C_{1}$ and $C_{2}$ are 1 to 2, but 2 is the most appropriate in many cases. Appropriate values for $\omega_{\min }$ and $\omega_{\max }$ are 0.4 and 0.9 (Eberhart et al, 2000) respectively

\subsection{Objective Function:}

The main objective is to minimize the total power loss as given in eq. (1) while meeting the following constraints.

- The network power flow equation must be satisfied.

- The voltage at every bus in the network should be within the acceptable range (Utility's standard ANSI Std. C84.1-1989) i.e., within permissible limit ( $\pm 5 \%$ ) (Wills, 2004),

$$
\mathrm{V}_{\text {min }} \leq \mathrm{V}_{\mathrm{i}} \leq \mathrm{V}_{\max } \forall_{i} \in\{\text { buses of the network\} }
$$

\subsection{PSO Procedure:}

The PSO-based approach for solving the optimal placement of DG problem to minimize the loss takes the following steps:

Step 1: Input line and bus data, and bus voltage limits.

Step 2: Calculate the loss using distribution load flow based on backward sweep-forward sweep method.

Step 3: Randomly generates an initial population (array) of particles with random positions and velocities on dimensions (Size of DGs and Location of DGs) in the solution space. Set the iteration counter $k=0$.

Step 4: For each particle if the bus voltage is within the limits as given above, evaluate the total loss in equation (1). Otherwise, that particle is infeasible.

Step 5: For each particle, compare its objective value with the individual best. If the objective value is lower than Pbest, set this value as the current Pbest, and record the corresponding particle position.

Step 6: Choose the particle associated with the minimum individual best Pbest of all particles, and set the value of this Pbest as the current overall best Gbest.

Step 7: Update the velocity and position of particle using (6) and (7) respectively.

Step 8: If the iteration number reaches the maximum limit, go to Step 9. Otherwise, set iteration index $k=k+1$, and go back to Step 4.

Step 9: Print out the optimal solution to the target problem. The best position includes the optimal locations and size of DG and the corresponding fitness value representing the minimum total real power loss.

\section{Test system}

This methodology is tested on test system contains 33 buses and 32 branches as shown in fig.5. It is a radial system with a total load of 3.72 MW and 2.3 MVAR (Kashem et al, 2000). A computer program is written in MATLAB 7 to find the optimal size of DG at various buses and approximate total loss with DG at various locations to find out the best location by analytical method, repeated load flow (Acharya et al, 2006) and PSO. 


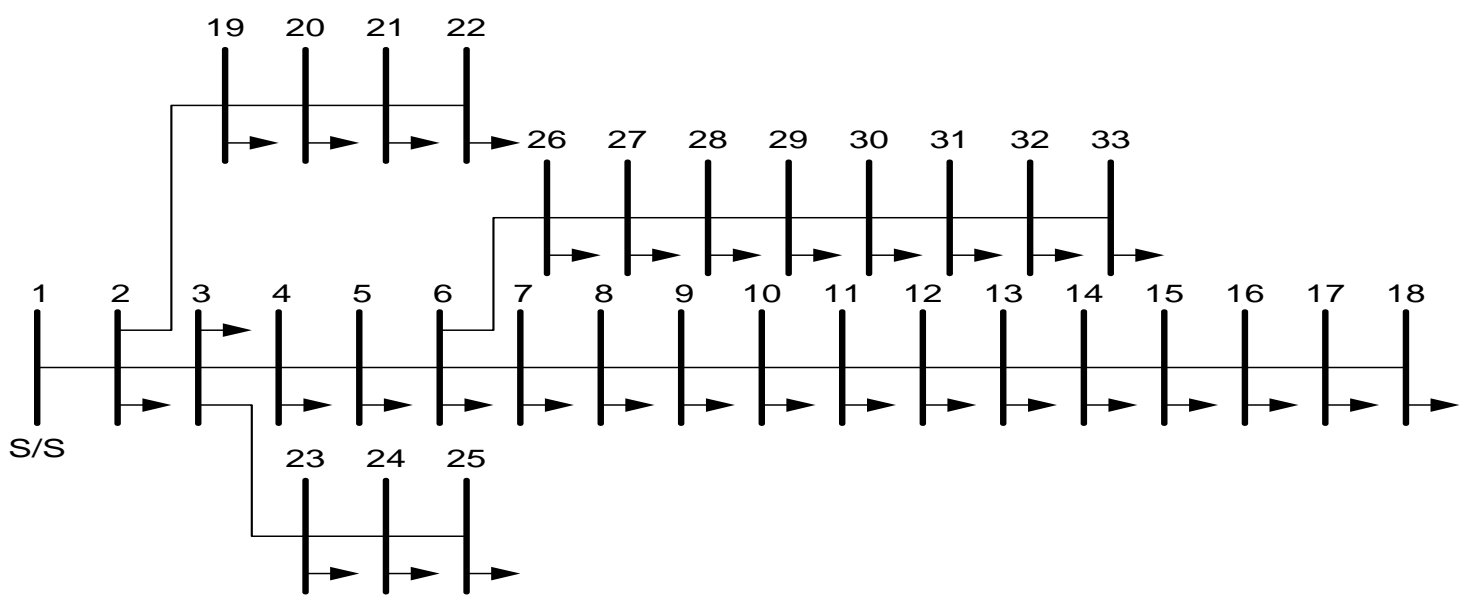

Figure 5. Single line diagram of 33 bus distribution test system.

\section{Results and Discussions}

Based on the analytical expression, the optimum size of DG is calculated at each bus for the test system and bus having least total power loss will be the optimal location for the placement of DG; the best location is bus 6 with a total power loss of 111.2 $\mathrm{kW}$, but this approach violates the voltage limits as shown in fig.(6). The optimal placement of DG by loss sensitivity approach is not able to identify the best location. The optimal placement of DG by repeated load flow with loss of $111.02 \mathrm{~kW}$ as shown in Table I violate the voltage limits, If voltage limits are taken into consideration then size of DG will increase but if the same is done by PSO technique by taking the voltage limit constraints into consideration the size of DG will decrease drastically i.e. $240 \mathrm{~kW}$, with approximately same power loss as shown in table II, and voltage profile is as shown in fig.(7).

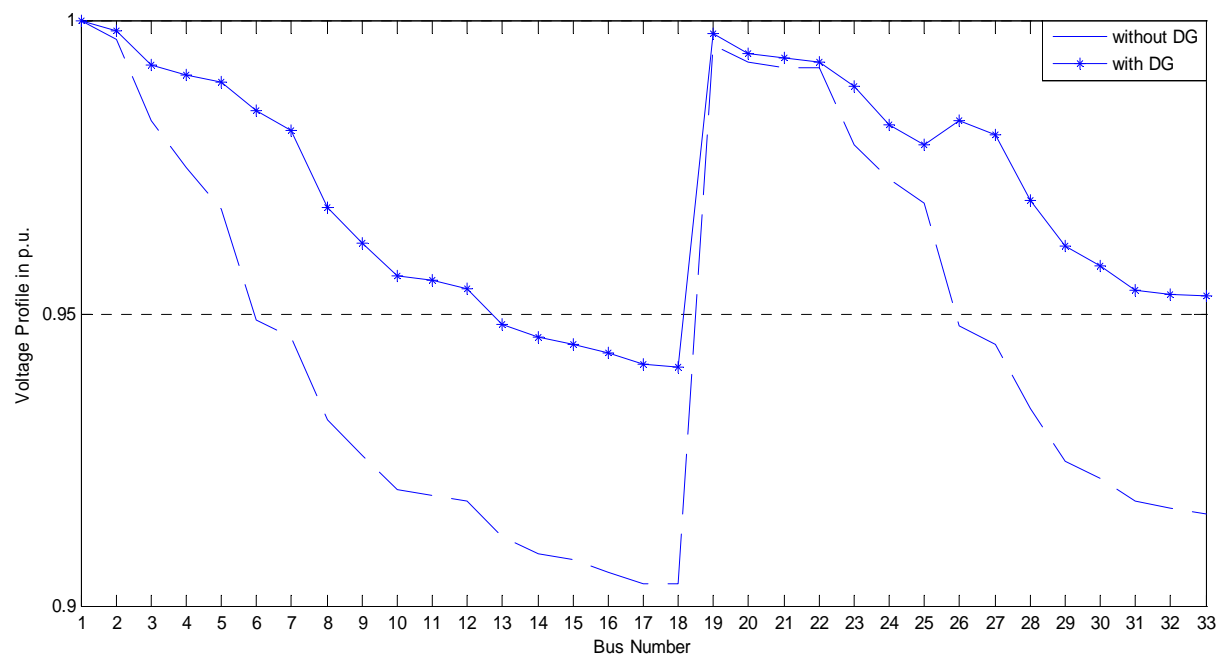

Figure 6. Variation of voltage profile by analytical method. 
Table I Power loss with and without DG for 33 bus system without limits

\begin{tabular}{|c|c|c|c|c|}
\hline \multirow{2}{*}{ Method } & \multirow{2}{*}{ Optimum location } & \multirow{2}{*}{ Optimum DG size (MW) } & \multicolumn{2}{|c|}{ Power loss (KW) } \\
\cline { 4 - 5 } & Bus 6 & 2.49 & 210.97 & 111.2 \\
\hline Analytical approach & Bus 10 & 1.4 & 210.97 & 123.72 \\
\hline Loss sensitivity factor & Bus 6 & 2.59 & 210.97 & 111.02 \\
\hline Repeated load flow & \multicolumn{3}{|c}{} \\
\hline
\end{tabular}

Table II Power loss with and without DG for 33 bus system with limits

\begin{tabular}{|c|c|c|c|c|}
\hline \multirow{2}{*}{ Method } & \multirow{2}{*}{ Optimum location } & Optimum DG size (MW) & \multicolumn{2}{|c|}{ Power loss (KW) } \\
\cline { 4 - 5 } & & Without DG & With DG \\
\hline Repeated load flow & Bus 6 & 3.15 & 210.97 & 115.2 \\
\hline PSO & Bus 7 & 2.91 & 210.97 & 115.1 \\
\hline
\end{tabular}

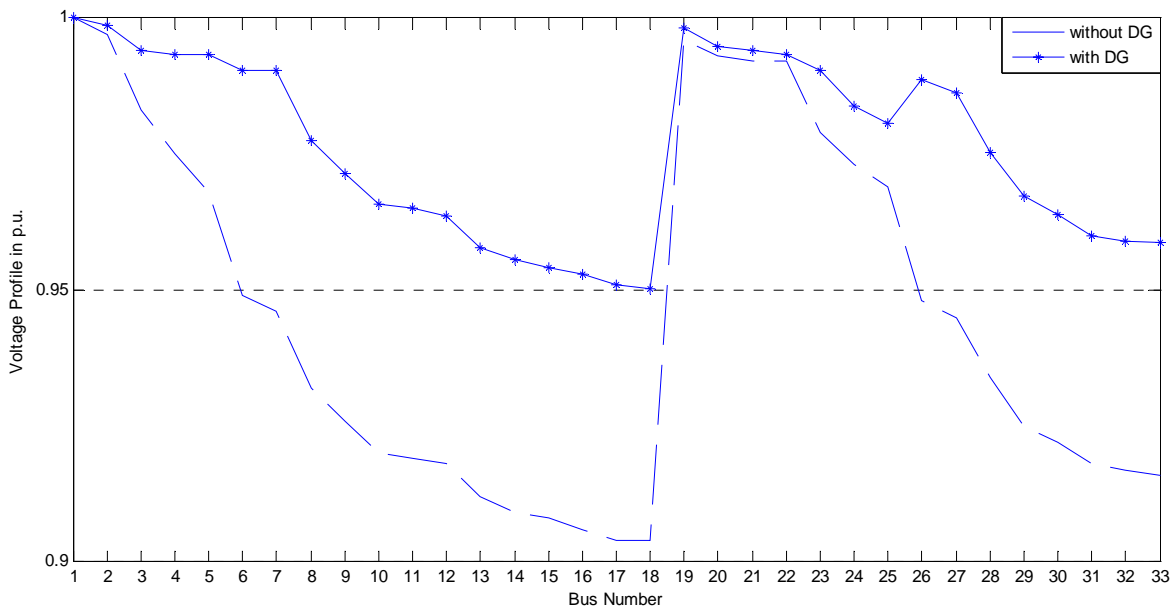

Figure 7. Variation of voltage profile by PSO.

\section{Conclusion}

Optimal placement of DG plays an important role for maximizing the total real power loss reduction in the distribution system with active power compensation. The optimal placement by analytical method violates the line voltage limits, if voltage is within limits then the size and line losses increases. The optimal placement of DG by PSO technique taking the voltage limits of the system into consideration to minimizing the real power loss improves the results drastically. But in practice the best location or size may not always be possible due to many constraints i.e. such size may not be available in the market.

\section{References}

Acharya N., Mahat P., Mithulananthan N., 2006. An analytical approach for DG allocation in primary distribution network, Electric Power \& Energy Systems, Vol.28, No.10, pp.669-678, December.

Ackermann T., Andersson G., and Solder L., 2001. Distributed generation: a definition, Electric Power system Research, Vol.57, No.3, pp.195-204, April.

CIGRE, 1999. Impact of increasing contribution of dispersed generation on the power system, Working Group37.23..

Eberhart R.C.and Shi Y., 2000. Comparing inertial weights and constriction factor in particle swarm optimization, Proceedings of the International Congress on Evaluating Computation, San Diego,Calfornia, IEEE service center, Piscataway, NJ, pp 84-88. 
Elgerd I.O. 1971. Electric energy system theory: an introduction, McGraw-Hill.

Ghosh S., Ghoshal S.P., Ghosh S., 2010. Optimal sizing and placement of distributed generation in a network system, Electric Power and Energy Systems, Vol.32, pp. 849-856, January.

Haque M.H., 1996. Efficient load flow method for distribution systems with radial or mesh configuration, IEE Proceedings Generation, Transmission and Distribution, Vol. 143, No. 1, pp.33-38, January.

Illerhaus S.W., Versteg J.S., 2000. Optimal operation of industrial CHP-based power systems in liberalized energy markets, IEEE Industry Applications Conference, Vol. 2, pp. 901-908.

Kashem M.A., Ganapathy V., Jasmon G.B., Buhari M.I., 2000. A novel method for loss minimization in distribution networks, Int.Conference on Electric Utility Deregulation and Restructuring and Power Technology, London, April.

Kazemi A., Sadeghi M., 2009. Sitting and sizing of distributed generation for loss reduction, Power and Energy Conference, APPEEC, pp.1-4, Asia-Pacific, Wuhan.

Kennedy J., Eberhart R., 1995. Particle Swarm Optimizer, IEEE International Conference on Neural Networks , Perth(Australia), IEEE Service Centre Piscataway, NJ, IV, pp. 1942-1948.

Khoa T.Q.D., Binh P.T.T., Tran H.B., 2006. Optimizing location and sizing of distributed generation in distribution systems Proceedings of IEEE PES Power Systems Conference and Exposition-PSCE 2006, pp. 725-732, October/November.

Lakshami Devi A., Subramanyam B., 2008. Sitting of DG unit operated at optimal power factor to reduce losses in radial distribution system. A case study, Theoretical and Applied Information Technology.

Wills H.L., 2004. Power Distribution Planning Reference Book. New York: Marcel Deckker.

Biographical notes

Satish Kansal received the M.E. degree in Power System Engineering from the Punjab Engineering College (PEC), Chandigarh, in 1998.Currently, he is pursuing Ph.D. at IIT Roorkee (U.K.) and is a Associate Professor in the Electrical Engineering Department at BHSBIET Lehragaga (Pb.). His research interest includes power distribution system, power system optimization, distributed generation and renewable energy.

B.Bhaskara Rama Sai received the B.Tech. degree in Electrical and Electronics Engg. From MVGR college of Engg., Vizianagaram (A.P.). Currently he is pursuing M-Tech at IIT Roorkee in System Engineering and Operation Research.

Barjeev Tyagi received the PhD in Electrical Engineering, IIT-Kanpur, 2005 and M. Tech Electrical Engineering (Control System) from IIT-Kharagpur in the year 2000. Prior to these he completed his B. E. Electrical Engineering from IIT-Roorkee (Formally Univ. of Roorkee) in 1987. Presently he is serving as Assistant Professor in EED, IIT-Roorkee since 2007. His research interests include power system deregulation, power system optimization, distributed generation and control.

Vishal kumar received the Ph.D.degree in power system engineering from Indian Institute of Technology, Roorkee (IITR), India, in 2007. Currently he is faculty member in the Department of Electrical Engineering, Indian Institute of Technology, Roorkee. His research interest includes power distribution system, operation and control, digital design and verification.

Received January 2011

Accepted March 2011

Final acceptance in revised form April 2011 